\title{
Generalized Lichen Planus-like Eruption Related to Trimebutine
}

\author{
Dimitra Koumaki ${ }^{1}$, Vasiliki Koumaki ${ }^{2}$, Alexander Katoulis ${ }^{3}$, Sotirios Boumpoucheropoulos ${ }^{4}$, \\ George Evangelou $^{1}$, Maria Stefanidou ${ }^{1}$, Konstantinos Krasagakis ${ }^{1}$ \\ ${ }^{1}$ Dermatology Department, University Hospital of Heraklion, Heraklion, Crete, Greece \\ 2 Microbiology Department, Medical School of Athens, Athens, Greece \\ ${ }^{3}$ 2nd Department of Dermatology and Venereology, National and Kapodistrian University of Athens, Medical School, "Attikon" General University Hospital, \\ Athens, Greece \\ ${ }^{4}$ Medical Oncology Department, Agioi Anargyroi General Oncological Hospital of Kifissia, Athens, Greece
}

\section{Doi: 10.12890/2020_002103 - European Journal of Case Reports in Internal Medicine - @ EFIM 2020}

Received: $27 / 10 / 2020$

Accepted: $28 / 10 / 2020$

Published: $24 / 11 / 2020$

How to cite this article: Koumaki D, Koumaki V, Katoulis A, Boumpoucheropoulos S, Evangelou G, Stefanidou M, Krasagakis K. Generalized lichen planuslike eruption related to trimebutine. EJCRIM 2020;7: doi:10.12890/2020_002103.

Conflicts of Interests: The Authors declare that there are no competing interests.

This article is licensed under a Commons Attribution Non-Commercial 4.0 License

\section{ABSTRACT}

Trimebutine is a spasmolytic agent with antimuscarinic effects that is used for the treatment of irritable bowel syndrome (IBS) and lower gastrointestinal tract motility disorders. Lichenoid drug eruptions (LDE) to trimebutine maleate have not been previously reported. Here we present the case of a 50-year-old male patient who developed an extensive lichenoid eruption on his upper and lower extremities and trunk 4 weeks after starting treatment with trimebutine maleate $300 \mathrm{mg}$ once daily for IBS. Two months after discontinuation of the drug and administration of topical treatment with emollients and corticosteroids, the LDE cleared completely with no recurrence. The diagnosis of LDE due to trimebutine was made, based upon the clinical features resembling lichen planus, the histological findings of interface dermatitis, the evidence of a temporal relationship between drug intake and the development of skin lesions, and resolution upon discontinuation of the drug. To the best of the authors' knowledge, LDE following trimebutine maleate intake has not been previously reported. Management of trimebutine-induced LDE includes withdrawal of the causative agent and treatment with potent topical corticosteroids.

\section{LEARNING POINTS}

- Cutaneous adverse events due to trimebutine maleate, an antispasmodic agent frequently used for the treatment of irritable bowel syndrome (IBS), have rarely been reported.

- Lichenoid drug eruption (LDE), also called drug-induced lichen planus, is an uncommon cutaneous adverse effect of several drugs.

- Here we report the first case of trimebutine maleate-induced LDE.

\section{KEYWORDS}

Trimebutine maleate, lichenoid drug eruption, lichen planus, cutaneous adverse reaction

\section{INTRODUCTION}

Lichenoid drug eruption (LDE), also called drug-induced lichen planus, is an uncommon cutaneous adverse effect of several drugs ${ }^{[1-3]}$. The length of time between initiation of the offending drug and the appearance of cutaneous lesions varies from months to a year or more and depends upon the class of drug, dose, host reaction and concurrent medications. Trimebutine maleate is a spasmolytic that has been extensively used for the treatment of irritable bowel syndrome (IBS) ${ }^{[4]}$. Here we report the first case of LDE following trimebutine treatment. 


\section{CASE DESCRIPTION}

A 50-year-old male patient presented with a 2-month history of a severely pruritic generalized eruption extending over the extremities. Examination showed symmetric eruption of discrete violaceus polygonal papules on the dorsal aspects of the upper and lower extremities that had spread to the trunk and developed into a diffuse eruption with some lichenification (Figs. 1 and 2). Oral mucosa was normal. Nails were not involved. The patient's medical history showed he had gastroesophageal reflux disease (GERD) and had been started on treatment with trimebutine $300 \mathrm{mg}$ once daily 4 weeks before the skin eruption had started. Histology of a skin biopsy taken from the right forearm revealed epidermal hyperplasia with hyperkeratotic foci, focal interruption of the granular layer, lichenoid dermatitis, band-like lymphocytic infiltrate in the papillary dermis, exocytosis of lymphoid cells into the upper epidermis, and the presence of eosinophils in keeping with LDE.

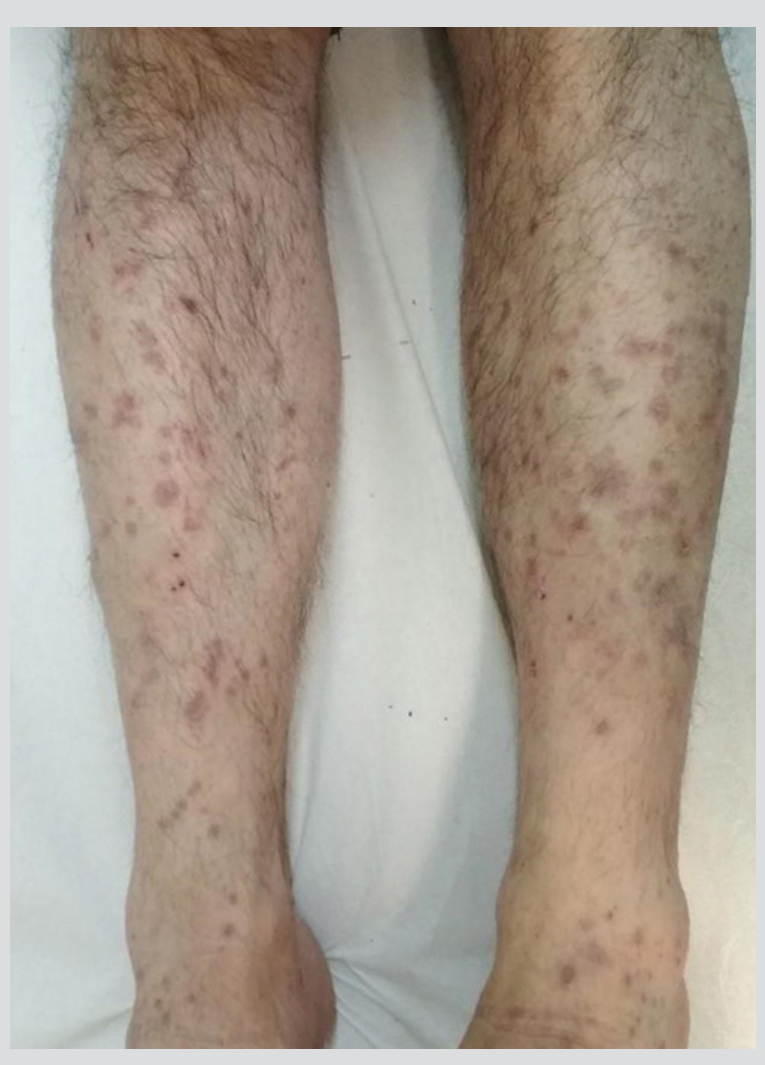

Figure 1. Discrete violaceus polygonal papules on the legs of the patient

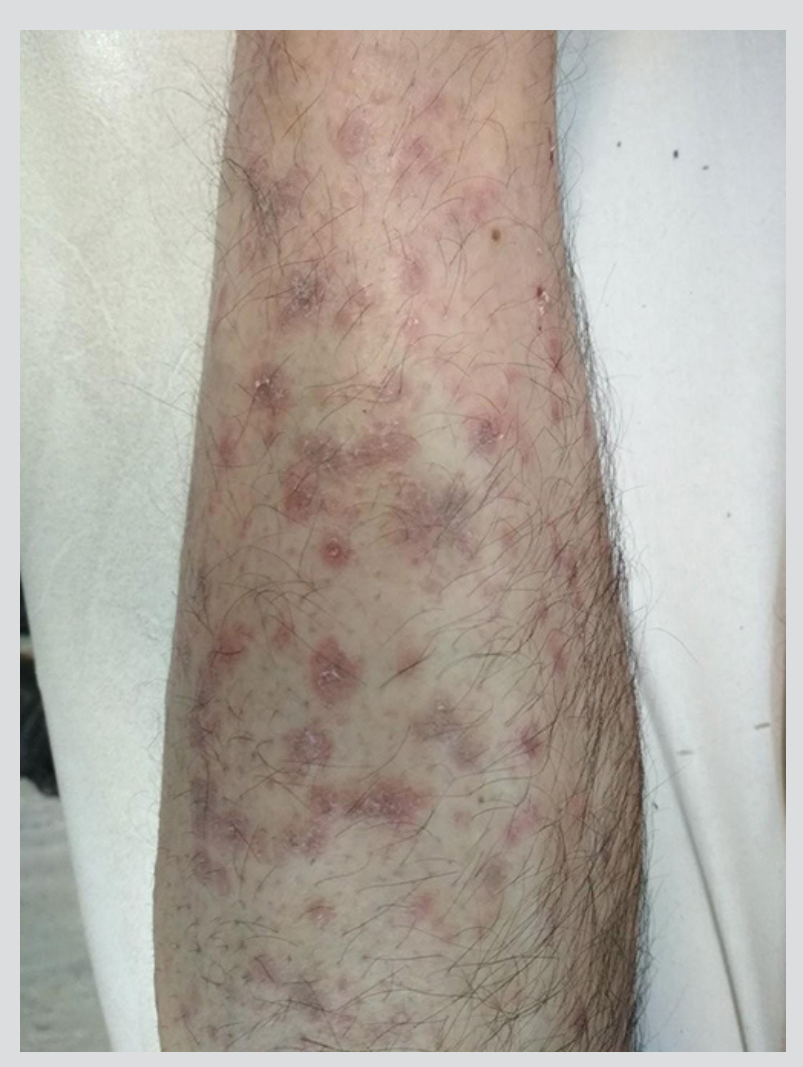

Figure 2. Lichenoid eruption on the forearms of the patient

A diagnosis of a drug-related lichenoid reaction as a result of trimebutine was made and the medication was withdrawn. The patient was prescribed topical emollients to be applied twice daily for 4 weeks, clobetasol propionate $0.05 \%$ ointment once daily for 4 weeks, and oral levocetirizine $5 \mathrm{mg}$ once daily for 4 weeks. No new lesions developed and the existing lesions cleared over a period of 2 months.

\section{DISCUSSION}

LDE, also called drug-induced lichen planus, is an uncommon cutaneous adverse effect of several drugs such as angiotensin-converting enzyme (ACE) inhibitors, thiazide diuretics, antimalarials, beta-blockers, gold salts, penicillamine and checkpoint inhibitors ${ }^{[1-3]}$.

LDE is characterized by a symmetric eruption of flat-topped, erythematous or violaceous papules, often grouped and confluent, on the trunk and extremities. Lesions closely resemble those seen in lichen planus, although in LDE the individual lesions are less monomorphic and may have eczematous or psoriasiform morphology with marked desquamation. In some patients, the lesions are localized to photoexposed areas and exhibit photoaggravation. In contrast to lichen planus, the LDE lesions are distributed on the extensor aspects of the extremities and dorsal aspects of the hands ${ }^{[2]}$.

The pathogenetic mechanism of lichenoid drug reaction is incompletely understood. T-cells, keratinocytes, dendritic cells and endothelial cells, which express activation markers, costimulatory molecules and adhesion molecules (e.g., MHC class II molecules, lymphocyte function- 
associated antigen (LFA) 1, L-selectin, intercellular adhesion molecule (ICAM) 1), are thought to be involved in the inflammatory reaction that ultimately leads to the apoptosis of basal keratinocytes ${ }^{[5,6]}$. The latency period between drug initiation and the appearance of the eruption varies from months to a year or more and depends upon the type of drug, dose, host reaction, previous exposure to the drug, and concurrent medications ${ }^{[1]}$. The diagnosis of LDE is based upon a combination of the following findings: clinical features consisting of symmetric eruption of flat-topped, erythematous or violaceous papules, often grouped and confluent, resembling lichen planus on the trunk and extremities, histological findings of interface dermatitis with a band-like lymphocytic infiltrate, evidence of a temporal relationship between drug intake and the development of skin lesions, and resolution upon drug withdrawal and recurrence upon rechallenge. LDE generally resolves spontaneously a few weeks to a few months after discontinuation of the offending drug.

To the best of our knowledge, we have reported the first case of LDE after trimebutine treatment. Trimebutine maleate, a widely prescribed medication for functional gastrointestinal disorders, is considered a generally well-tolerated drug with rare cutaneous side effects ${ }^{[3]}$. The new onset of lichenoid dermatitis in an individual receiving therapy with trimebutine should raise concern that this skin eruption may be associated with the medication. We recommend the withdrawal of the causative agent and topical treatment with extra-potent steroids such as clobetasol propionate $0.05 \%$ ointment.

\section{REFERENCES}

1. Brauer J, Votava HJ, Meehan S, Soter NA. Lichenoid drug eruption. Dermatol Online J 2009;15(8):13.

2. Asarch A, Gottlieb AB, Lee J, Masterpol KS, Scheinman PL, Stadecker MJ, et al. Lichen planus-like eruptions: an emerging side effect of tumor necrosis factor-alpha antagonists. J Am Acad Dermatol 2009;61(1):104-111.

3. Fox GN, Harrell CC, Mehregan DR. Extensive lichenoid drug eruption due to glyburide: a case report and review of the literature. Cutis 2005;76(1):41-45.

4. Salvioli B. Trimebutine: a state-of-the-art review. Minerva Gastroenterol Dietol 2019;65(3):229-238.

5. Yawalkar N, Egli F, Hari Y, Nievergelt H, Braathen LR, Pichler WJ. Infiltration of cytotoxic T cells in drug-induced cutaneous eruptions. Clin Exp Allergy 2000;30(6):847-855.

6. Yawalkar N, Shrikhande M, Hari Y, Nievergelt H, Braathen LR, Pichler WJ. Evidence for a role for IL-5 and eotaxin in activating and recruiting eosinophils in drug-induced cutaneous eruptions. J Allergy Clin Immunol 2000;106(6):1171-1176. 